Research Article

\title{
Existence of Two Positive Periodic Solutions for a Neutral Multi-Delay Logarithmic Population Model with a Periodic Harvesting Rate
}

\author{
Hui Fang \\ Department of Mathematics, Kunming University of Science and Technology, Yunnan 650500, China \\ Correspondence should be addressed to Hui Fang, kmustfanghui@hotmail.com \\ Received 14 July 2012; Accepted 12 November 2012 \\ Academic Editor: Wing-Sum Cheung \\ Copyright (c) 2012 Hui Fang. This is an open access article distributed under the Creative \\ Commons Attribution License, which permits unrestricted use, distribution, and reproduction in \\ any medium, provided the original work is properly cited. \\ By using an abstract existence result based on a coincidence degree theory for $k$-set contractive \\ mapping, a new result is obtained for the existence of at least two positive periodic solutions for \\ a neutral multidelay logarithmic population model with a periodic harvesting rate. An example is \\ given to illustrate the effectiveness of the result.
}

\section{Introduction}

In recent years, many papers have been published on the existence of positive periodic solutions for neutral delay logarithmic population models by using a topological degree theory for $k$-set contractive mapping (see, e.g., [1-5]). Recently, Xia [6] obtained some new sufficient conditions for the existence and uniqueness of an almost periodic solution of a multispecies logarithmic population model with feedback controls. However, few papers deal with the existence of multiple positive periodic solutions for neutral multidelay logarithmic population models with harvesting. The main difficulty is hard to obtain a priori bounds on solutions for neutral multi-delay models with harvesting.

In this paper, we consider the following neutral multi-delay logarithmic population model of single-species population growth with a periodic harvesting rate

$$
\frac{d y(t)}{d t}=y(t)\left[a(t)-\sum_{j=1}^{n} b_{j}(t) \ln y\left(t-\sigma_{j}(t)\right)-\sum_{i=1}^{m} c_{i}(t) \frac{d}{d t} \ln y\left(t-\tau_{i}(t)\right)\right]-h(t),
$$


where $a(t), b_{j}(t), \sigma_{j}(t)(j=1,2, \ldots, n), c_{i}(t), \tau_{i}(t)(i=1,2, \ldots, m), h(t)$ are nonnegative continuous T-periodic functions, and $h(t)$ denotes the harvesting rate. When $h(t) \equiv 0,(1.1)$ was considered by [2-5]. When $n=m=1, h(t) \equiv 0$, and $\sigma_{1}(t), \tau_{1}(t)$ are constants, (1.1) was considered by [7].

The purpose of this paper is to establish the existence of at least two positive periodic solutions for a neutral multi-delay logarithmic population model (1.1) by using a coincidence degree theory for $k$-set contractions. Motivated by the work of Chen [8], some novel techniques are employed to find a priori bounds on solutions.

\section{Preliminaries}

We now briefly state the part of the coincidence degree theory for $k$-set contractive mapping developed by Hetzer $[9,10]$. For more details, we refer to [11].

Let $Z$ be a Banach space. For a bounded subset $A \subset Z$, let $\Gamma_{Z}(A)$ denote the (Kuratowski) measure of noncompactness defined by

$$
\Gamma_{Z}(A)=\inf \left\{\delta>0: \exists \text { a finite number of subsets } A_{i} \subset A, A=\bigcup_{i} A_{i}, \operatorname{diam}\left(A_{i}\right) \leq \delta\right\} \text {. }
$$

Here, diam $\left(A_{i}\right)$ denotes the maximum distance between the points in the set $A_{i}$.

Let $X$ and $Z$ be Banach spaces with norms $\|\cdot\|_{X}$ and $\|\cdot\|_{Z}$, respectively and $\Omega$ a bounded open subset of $X$. A continuous and bounded mapping $N: \bar{\Omega} \rightarrow Z$ is called $k$-set contractive if for any bounded $A \subset \bar{\Omega}$, we have

$$
\Gamma_{Z}(N(A)) \leq k \Gamma_{X}(A) .
$$

Also, for a continuous and bounded map $T: X \rightarrow Y$, we define

$$
l(T)=\sup \left\{r \geq 0: \forall \text { bounded subset } A \subset X, r \Gamma_{X}(A) \leq \Gamma_{Y}(T(A))\right\} .
$$

Let $L: \operatorname{dom} L \subset X \rightarrow Z$ be a linear mapping and $N: X \rightarrow Z$ be a continuous mapping. The mapping $L$ will be called a Fredholm mapping of index zero if $\operatorname{dim} \operatorname{Ker} L=$ codim $\operatorname{Im} L<+\infty$ and $\operatorname{Im} L$ is closed in $Z$. If $L$ is a Fredholm mapping of index zero, there then exist continuous projectors $P: X \rightarrow X$ and $Q: Z \rightarrow Z$ such that $\operatorname{Im} P=\operatorname{Ker} L, \operatorname{Im} L=$

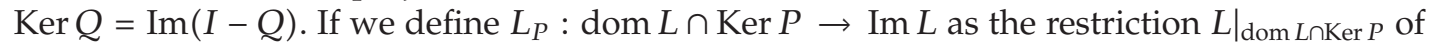
$L$ to dom $L \cap \operatorname{Ker} P$, then $L_{P}$ is invertible. We denote the inverse of that map by $K_{P}$. If $\Omega$ is an open bounded subset of $X$, the mapping $N$ will be called $L$ - $k$-set contractive on $\bar{\Omega}$ if $Q N(\bar{\Omega})$ is bounded and $K_{P}(I-Q) N: \bar{\Omega} \rightarrow X$ is $k$-set contractive. Since $\operatorname{Im} Q$ is isomorphic to Ker $L$, there exists an isomorphism $J: \operatorname{Im} Q \rightarrow \operatorname{Ker} L$.

Lemma 2.1 ([11, Proposition XI.2.]). Let $L$ be a closed Fredholm mapping of index zero and let $N: \bar{\Omega} \rightarrow Z$ be $k^{\prime}$-set contractive with

$$
0 \leq k^{\prime}<l(L)
$$

Then $N: \bar{\Omega} \rightarrow Z$ is a $L-k$-set contraction with constant $k=k^{\prime} / l(L)<1$. 
The following lemma [[11, page 213] will play a key role in this paper.

Lemma 2.2. Let $L$ be a Fredholm mapping of index zero and let $N: \bar{\Omega} \rightarrow Z$ be $L$-k-set contractive on $\bar{\Omega}, k<1$. Suppose

(i) $L x \neq \lambda N x$ for every $x \in \operatorname{dom} L \cap \partial \Omega$ and every $\lambda \in(0,1)$;

(ii) $Q N x \neq 0$ for every $x \in \partial \Omega \cap \operatorname{Ker} L$;

(iii) Brouwer degree $\operatorname{deg}_{B}(J Q N, \Omega \cap \operatorname{Ker} L, 0) \neq 0$.

Then $L x=N x$ has at least one solution in $\operatorname{dom} L \cap \bar{\Omega}$.

\section{Main Result}

Let $C_{T}^{0}$ denote the linear space of real valued continuous $T$-periodic functions on $R$. The linear space $C_{T}^{0}$ is a Banach space with the usual norm for $x \in C_{T}^{0}$ given by $|x|_{0}=\max _{t \in R}|x(t)|$. Let $C_{T}^{1}$ denote the linear space of $T$-periodic functions with the first-order continuous derivative. $C_{T}^{1}$ is a Banach space with norm $|x|_{1}=\max \left\{|x|_{0},\left|x^{\prime}\right|_{0}\right\}$.

Let $X=C_{T}^{1}$ and $Z=C_{T}^{0}$ and let $L: X \rightarrow Z$ be given by $L x=d x / d t$. Since $|L x|_{0}=$ $\left|x^{\prime}\right|_{0} \leq|x|_{1}$, we see that $L$ is a bounded (with bound $=1$ ) linear map.

Under the transformation of $y(t)=e^{x(t)},(1.1)$ can be rewritten as

$$
x^{\prime}(t)=a(t)-\sum_{j=1}^{n} b_{j}(t) x\left(t-\sigma_{j}(t)\right)-\sum_{i=1}^{m} c_{i}(t)\left(1-\tau_{i}^{\prime}(t)\right) x^{\prime}\left(t-\tau_{i}(t)\right)-\frac{h(t)}{e^{x(t)}}
$$

Next define a nonlinear map $N: X \rightarrow Z$ by

$$
N(x)(t)=a(t)-\sum_{j=1}^{n} b_{j}(t) x\left(t-\sigma_{j}(t)\right)-\sum_{i=1}^{m} c_{i}(t)\left(1-\tau_{i}^{\prime}(t)\right) x^{\prime}\left(t-\tau_{i}(t)\right)-\frac{h(t)}{e^{x(t)}} .
$$

Now, if $L x=N x$ for some $x \in X$, then the problem (3.1) has a $T$-periodic solution $x(t)$.

In the following, we denote

$$
\bar{g}=\frac{1}{T} \int_{0}^{T} g(t) d t, \quad g^{l}=\min _{t \in[0, T]} g(t), \quad g^{u}=\max _{t \in[0, T]} g(t),
$$

where $g(t)$ is a continuous nonnegative $T$-periodic solution.

From now on, we always assume that

$\left(H_{1}\right) a(t), b_{j}(t) \in C(R,(0,+\infty)), \sigma_{j}(t), c_{i}(t), \tau_{i}(t) \in C^{1}(R,(0,+\infty))$, for all $j \in\{1,2, \ldots$, $n\}$, for all $i \in\{1,2, \ldots, m\}$.

$\left(H_{2}\right) \quad \sigma_{j}^{\prime}(t)<1, \tau_{i}^{\prime}(t)<1$, for all $t \in R$, and

$$
\Gamma(t)=\sum_{j=1}^{n} \frac{b_{j}\left(\mu_{j}(t)\right)}{1-\sigma_{j}^{\prime}\left(\mu_{j}(t)\right)}-\sum_{i=1}^{m} \frac{c_{i}^{\prime}\left(\gamma_{i}(t)\right)}{1-\tau_{i}^{\prime}\left(\gamma_{i}(t)\right)}>0,
$$


where $\mu_{j}(t)$ is the inverse function of $t-\sigma_{j}(t), \gamma_{i}(t)$ is the inverse function of $t-\tau_{i}(t)$, for all $j \in$ $\{1,2, \ldots, n\}$, for all $i \in\{1,2, \ldots, m\}$.

$\left(H_{3}\right)$ Let

$$
1+\ln \frac{h^{l}}{\Gamma^{u}}<\frac{a^{u}}{\Gamma^{l}} \leq \frac{h^{l}}{\Gamma^{u}}<1
$$

$\left(H_{4}\right)$ Let

$$
\begin{gathered}
T\left(\bar{\Gamma}+\sum_{j=1}^{n} \bar{b}_{j}\right)+\sum_{i=1}^{m} c_{i}^{u}<1, \\
\max \left\{1+\ln \frac{h^{u}}{\sum_{j=1}^{n} b_{j}^{l}}, 0\right\}<\frac{a^{l}-\sum_{i=1}^{m} c_{i}^{u}\left(1-\tau_{i}^{\prime}\right)^{u} M_{0}}{\sum_{j=1}^{n} b_{j}^{u}}<R_{0}
\end{gathered}
$$

where

$$
\begin{gathered}
M_{0}=\frac{a^{u}+\sum_{j=1}^{n} b_{j}^{u} R_{0}+h^{u} e^{R_{0}}}{1-\sum_{i=1}^{m} c_{i}^{u}\left(1-\tau_{i}^{\prime}\right)^{u}}, \quad R_{0}=q+p, \\
q=2\left|\ln \frac{h^{l}}{\Gamma^{u}}\right|, \quad p=\frac{2 T \bar{a}+\left(\bar{\Gamma}+\sum_{j=1}^{n} \bar{b}_{j}\right) T q}{1-T\left(\bar{\Gamma}+\sum_{j=1}^{n} \bar{b}_{j}\right)-\sum_{i=1}^{m} c_{i}^{u}} .
\end{gathered}
$$

We first give some technological lemmas.

Set

$$
f(x)=d-x-r e^{-x}, \quad x \in(-\infty,+\infty) .
$$

Lemma 3.1. Assume that $d, r$ are positive constants such that

$$
1+\ln r<d
$$

Then there exist $x_{*}^{-}, x_{*}^{+}$such that

$$
\begin{gathered}
f\left(x_{*}^{-}\right)=f\left(x_{*}^{+}\right)=0, \\
x_{*}^{-}<\ln r<x_{*}^{+}<d, \\
f(x)>0 \quad \text { for } x \in\left(x_{*}^{-}, x_{*}^{+}\right) ; \quad f(x)<0 \quad \text { for } x \in\left(-\infty, x_{*}^{-}\right) \cup\left(x_{*}^{+},+\infty\right) .
\end{gathered}
$$

If one assumes further that

$$
d \leq r<1,
$$


then the following inequalities also hold:

$$
2 \ln r<x_{*}^{-}<\ln r<x_{*}^{+} \leq 0 .
$$

Proof. Clearly, $f^{\prime}(x)=r / e^{x}-1=0$ if and only if $x=\ln r$. Therefore, noticing that $\lim _{x \rightarrow \pm \infty} f(x)=-\infty$, we have

$$
\sup _{x \in(-\infty,+\infty)} f(x)=d-\ln r-1>0
$$

Set

$$
g(r)=2 \ln r+\frac{1}{r}-r
$$

Since

$$
g^{\prime}(r)=\frac{2}{r}-\frac{1}{r^{2}}-1=-\frac{(1-r)^{2}}{r^{2}}<0 \quad \text { for } 0<r<1,
$$

$g(r)$ is monotonically decreasing on $(0,1]$.

Therefore, we have

$$
g(r)>g(1)=0
$$

that is,

$$
2 \ln r+\frac{1}{r}>r
$$

which implies

$$
f(2 \ln r)=d-2 \ln r-\frac{1}{r}<d-r \leq 0 .
$$

Again, noticing that

$$
f(0)=d-r \leq 0,
$$

by the monotonicity of the function $f(x)$ on the interval $(-\infty, \ln r)$ and $(\ln r,+\infty)$, it is easy to see that the assertion holds. 
Set

$$
\begin{aligned}
F(x) & =\frac{a^{l}-\sum_{i=1}^{m} c_{i}^{u}\left(1-\tau_{i}^{\prime}\right)^{u} M_{0}}{\sum_{j=1}^{n} b_{j}^{u}}-x-\frac{h^{u}}{\sum_{j=1}^{n} b_{j}^{l}} e^{-x}, \\
G(x) & =\frac{\bar{a}}{\sum_{j=1}^{n} \bar{b}_{j}}-x-\frac{\bar{h}}{\sum_{j=1}^{n} \bar{b}_{j}} e^{-x}, \\
H(x) & =\frac{a^{u}+\sum_{i=1}^{m} c_{i}^{u}\left(1-\tau_{i}^{\prime}\right)^{u} M_{0}}{\sum_{j=1}^{n} b_{j}^{l}}-x-\frac{h^{l}}{\sum_{j=1}^{n} b_{j}^{u}} e^{-x} .
\end{aligned}
$$

Lemma 3.2. Assume that $\left(H_{1}\right),\left(H_{2}\right)$, and $\left(H_{4}\right)$ hold. Then the following assertions hold.

(1) There exist $u^{-}, u^{+}$such that

$$
\begin{gathered}
F\left(u^{-}\right)=F\left(u^{+}\right)=0, \\
u^{-}<\ln \frac{h^{u}}{\sum_{j=1}^{n} b_{j}^{l}}<u^{+}<\frac{a^{l}-\sum_{i=1}^{m} c_{i}^{u}\left(1-\tau_{i}^{\prime}\right)^{u} M_{0}}{\sum_{j=1}^{n} b_{j}^{u}}, \\
F(x)>0 \quad \text { for } x \in\left(u^{-}, u^{+}\right) ; \quad F(x)<0 \quad \text { for } x \in\left(-\infty, u^{-}\right) \cup\left(u^{+},+\infty\right) .
\end{gathered}
$$

(2) There exist $x^{-}, x^{+}$such that

$$
\begin{gathered}
G\left(x^{-}\right)=G\left(x^{+}\right)=0, \\
x^{-}<\ln \frac{\bar{h}}{\sum_{j=1}^{n} \bar{b}_{j}}<x^{+}<\frac{\bar{a}}{\sum_{j=1}^{n} \bar{b}_{j}},
\end{gathered}
$$

$G(x)>0$ for $x \in\left(x^{-}, x^{+}\right) ; \quad G(x)<0$ for $x \in\left(-\infty, x^{-}\right) \cup\left(x^{+},+\infty\right)$.

(3) There exist $l^{-}, l^{+}$such that

$$
\begin{gathered}
H\left(l^{-}\right)=H\left(l^{+}\right)=0, \\
l^{-}<\ln \frac{h^{l}}{\sum_{j=1}^{n} b_{j}^{u}}<l^{+}<\frac{a^{u}+\sum_{i=1}^{m} c_{i}^{u}\left(1-\tau_{i}^{\prime}\right)^{u} M_{0}}{\sum_{j=1}^{n} b_{j}^{l}}, \\
H(x)>0 \quad \text { for } x \in\left(l^{-}, l^{+}\right) ; \quad H(x)<0 \quad \text { for } x \in\left(-\infty, l^{-}\right) \cup\left(l^{+},+\infty\right) .
\end{gathered}
$$

(4)

$$
l^{-}<x^{-}<u^{-}<u^{+}<x^{+}<l^{+} .
$$


Proof. Noticing that

$$
\begin{gathered}
\frac{a^{l}-\sum_{i=1}^{m} c_{i}^{u}\left(1-\tau_{i}^{\prime}\right)^{u} M_{0}}{\sum_{j=1}^{n} b_{j}^{u}}<\frac{\bar{a}}{\sum_{j=1}^{n} \bar{b}_{j}}<\frac{a^{u}+\sum_{i=1}^{m} c_{i}^{u}\left(1-\tau_{i}^{\prime}\right)^{u} M_{0}}{\sum_{j=1}^{n} b_{j}^{l}} \\
\frac{h^{l}}{\sum_{j=1}^{n} b_{j}^{u}} \leq \frac{\bar{h}}{\sum_{j=1}^{n} \bar{b}_{j}} \leq \frac{h^{u}}{\sum_{j=1}^{n} b_{j}^{l}},
\end{gathered}
$$

we have

$$
F(x)<G(x)<H(x)
$$

It follows from $\left(H_{1}\right),\left(H_{2}\right)$, and $\left(H_{4}\right),(3.25)$ that

$$
\begin{gathered}
1+\ln \frac{h^{u}}{\sum_{j=1}^{n} b_{j}^{l}}<\frac{a^{l}-\sum_{i=1}^{m} c_{i}^{u}\left(1-\tau_{i}^{\prime}\right)^{u} M_{0}}{\sum_{j=1}^{n} b_{j}^{u}}, \\
1+\ln \frac{\bar{h}}{\sum_{j=1}^{n} \bar{b}_{j}}<\frac{\bar{a}}{\sum_{j=1}^{n} \bar{b}_{j}}, \\
1+\ln \frac{h^{l}}{\sum_{j=1}^{n} b_{j}^{u}}<\frac{a^{u}+\sum_{i=1}^{m} c_{i}^{u}\left(1-\tau_{i}^{\prime}\right)^{u} M_{0}}{\sum_{j=1}^{n} b_{j}^{l}} .
\end{gathered}
$$

Therefore, by Lemma 3.1, the assertions (1)-(3) hold. Furthermore, by (3.26) and the assertions (1)-(3), the assertion (4) also holds.

Lemma 3.3 (see [12]). L is a Fredholm map of index 0 and satisfies

$$
l(L) \geq 1
$$

Lemma 3.4 (see [2]). Suppose $\sigma \in C_{T}^{1}$ and $\sigma^{\prime}(t)<1$, for all $t \in[0, T]$. Then the function $t-\sigma(t)$ has an inverse function $\mu(t)$ satisfying $\mu \in C(R, R)$ with $\mu(a+T)=\mu(a)+T$.

Lemma 3.5. Assume that $\left(H_{1}\right)-\left(H_{4}\right)$ hold. Let $k_{0}=\sum_{i=1}^{m} c_{i}^{u}\left(1-\tau_{i}^{\prime}\right)^{u}$, and

$$
\Omega=\left\{\begin{array}{l|l}
x \in X & \begin{array}{l}
\max _{t \in[0, T]} x(t) \in\left(l^{-}-\delta, R_{0}+\delta\right), \\
\min _{t \in[0, T]} x(t) \in\left(l^{-}-\delta, l^{+}+\delta\right), \\
\max _{t \in[0, T]}\left|x^{\prime}(t)\right|<M_{0} .
\end{array}
\end{array}\right\}
$$

where $0<\delta<l^{-}$. Then $N: \bar{\Omega} \rightarrow Z$ is a $k_{0}$-set-contractive map.

Proof. The proof is similar to that of Lemma 3.3 in [12], so we omit it. 
Theorem 3.6. Assume that $\left(H_{1}\right)-\left(H_{4}\right)$ hold. Then (1.1) has at least two positive T-periodic solutions.

Proof. Let $L x=\lambda N x$ for $x \in X$, that is,

$$
x^{\prime}(t)=\lambda\left[a(t)-\sum_{j=1}^{n} b_{j}(t) x\left(t-\sigma_{j}(t)\right)-\sum_{i=1}^{m} c_{i}(t)\left(1-\tau_{i}^{\prime}(t)\right) x^{\prime}\left(t-\tau_{i}(t)\right)-\frac{h(t)}{e^{x(t)}}\right], \quad \lambda \in(0,1) .
$$

Therefore, we have

$$
x^{\prime}(t)=\lambda\left[a(t)-\sum_{j=1}^{n} b_{j}(t) x\left(t-\sigma_{j}(t)\right)-\sum_{i=1}^{m} c_{i}(t)\left[x\left(t-\tau_{i}(t)\right)\right]^{\prime}-\frac{h(t)}{e^{x(t)}}\right], \quad \lambda \in(0,1) .
$$

By (3.31), we have

$$
\left[x(t)+\lambda \sum_{i=1}^{m} c_{i}(t) x\left(t-\tau_{i}(t)\right)\right]^{\prime}=\lambda\left[a(t)-\sum_{j=1}^{n} b_{j}(t) x\left(t-\sigma_{j}(t)\right)+\sum_{i=1}^{m} c_{i}^{\prime}(t) x\left(t-\tau_{i}(t)\right)-\frac{h(t)}{e^{x(t)}}\right] .
$$

Integrating this identity leads to

$$
\int_{0}^{T}\left[\sum_{j=1}^{n} b_{j}(t) x\left(t-\sigma_{j}(t)\right)-\sum_{i=1}^{m} c_{i}^{\prime}(t) x\left(t-\tau_{i}(t)\right)+\frac{h(t)}{e^{x(t)}}\right] d t=\int_{0}^{T} a(t) d t .
$$

By Lemma 3.4, we have

$$
\mu_{j}(T+s)=T+\mu_{j}(s), \quad \gamma_{i}(T+s)=T+\gamma_{i}(s),
$$

where $t=\mu_{j}(s)$ is the inverse function of $s=t-\sigma_{j}(t)$, and $t=\gamma_{i}(s)$ is the inverse function of $s=t-\tau_{i}(t)$, for all $j \in\{1,2, \ldots, n\}$ and for all $i \in\{1,2, \ldots, m\}$.

Then

$$
\begin{aligned}
\int_{0}^{T} \sum_{j=1}^{n} b_{j}(t) x\left(t-\sigma_{j}(t)\right) d t & =\sum_{j=1}^{n} \int_{-\sigma_{j}(0)}^{T-\sigma_{j}(T)} b_{j}\left(\mu_{j}(s)\right) \frac{x(s)}{1-\sigma_{j}^{\prime}\left(\mu_{j}(s)\right)} d s \\
& =\sum_{j=1}^{n} \int_{0}^{T} \frac{b_{j}\left(\mu_{j}(s)\right)}{1-\sigma_{j}^{\prime}\left(\mu_{j}(s)\right)} x(s) d s, \\
\int_{0}^{T} \sum_{i=1}^{m} c_{i}^{\prime}(t) x\left(t-\tau_{i}(t)\right) d t & =\sum_{i=1}^{m} \int_{-\tau_{i}(0)}^{T-\tau_{i}(T)} c_{i}^{\prime}\left(\gamma_{i}(s)\right) \frac{x(s)}{1-\tau_{i}^{\prime}\left(\gamma_{i}(s)\right)} d s \\
& =\sum_{i=1}^{m} \int_{0}^{T} \frac{c_{i}^{\prime}\left(\gamma_{i}(s)\right)}{1-\tau_{i}^{\prime}\left(\gamma_{i}(s)\right)} x(s) d s .
\end{aligned}
$$


From (3.33)-(3.35), we have

$$
\int_{0}^{T}\left[a(s)-\left(\sum_{j=1}^{n} \frac{b_{j}\left(\mu_{j}(s)\right)}{1-\sigma_{j}^{\prime}\left(\mu_{j}(s)\right)}-\sum_{i=1}^{m} \frac{c_{i}^{\prime}\left(\gamma_{i}(s)\right)}{1-\tau_{i}^{\prime}\left(\gamma_{i}(s)\right)}\right) x(s)-\frac{h(s)}{e^{x(s)}}\right] d s=0
$$

which implies

$$
a(\eta)-\Gamma(\eta) x(\eta)-\frac{h(\eta)}{e^{x(\eta)}}=0
$$

for some $\eta \in[0, T]$.

Therefore, by $\left(H_{2}\right)$, we have

$$
\frac{a^{u}}{\Gamma^{l}}-x(\eta)-\frac{h^{l} / \Gamma^{u}}{e^{x(\eta)}} \geq 0
$$

By $\left(H_{3}\right)$ and Lemma 3.1, we have

$$
2 \ln \frac{h^{l}}{\Gamma^{u}}<x(\eta) \leq 0
$$

Set

$$
q:=2\left|\ln \frac{h^{l}}{\Gamma^{u}}\right|
$$

then we have

$$
|x(t)| \leq|x(\eta)|+\int_{0}^{T}\left|x^{\prime}(t)\right| d t \leq q+\int_{0}^{T}\left|x^{\prime}(t)\right| d t,
$$

which implies

$$
|x|_{0} \leq q+\int_{0}^{T}\left|x^{\prime}(t)\right| d t .
$$


It follows from (3.30) that

$$
\begin{aligned}
& \int_{0}^{T}\left|x^{\prime}(t)\right| d t \\
& \quad=\lambda \int_{0}^{T}\left|a(t)-\sum_{j=1}^{n} b_{j}(t) x\left(t-\sigma_{j}(t)\right)-\sum_{i=1}^{m} c_{i}(t)\left(1-\tau_{i}^{\prime}(t)\right) x^{\prime}\left(t-\tau_{i}(t)\right)-\frac{h(t)}{e^{x(t)}}\right| d t \\
& \quad \leq \lambda \int_{0}^{T}\left|a(t)-\sum_{j=1}^{n} b_{j}(t) x\left(t-\sigma_{j}(t)\right)-\sum_{i=1}^{m} c_{i}(t)\left(1-\tau_{i}^{\prime}(t)\right) x^{\prime}\left(t-\tau_{i}(t)\right)\right| d t+\int_{0}^{T} \frac{h(t)}{e^{x(t)}} d t .
\end{aligned}
$$

By (3.36) and $\left(\mathrm{H}_{2}\right)$, we have

$$
\int_{0}^{T} \frac{h(t)}{e^{x(t)}} d t \leq \int_{0}^{T} a(t) d t+\int_{0}^{T} \Gamma(t) d t|x|_{0}=T \bar{a}+T \bar{\Gamma}|x|_{0}
$$

By this and (3.43), we obtain

$$
\int_{0}^{T}\left|x^{\prime}(t)\right| d t \leq T\left[2 \bar{a}+\bar{\Gamma}|x|_{0}+\sum_{j=1}^{n} \bar{b}_{j}|x|_{0}\right]+\sum_{i=1}^{m} \int_{0}^{T}\left|c_{i}(t)\left(1-\tau_{i}^{\prime}(t)\right) x^{\prime}\left(t-\tau_{i}(t)\right)\right| d t
$$

Meanwhile,

$$
\sum_{i=1}^{m} \int_{0}^{T}\left|c_{i}(t)\left(1-\tau_{i}^{\prime}(t)\right) x^{\prime}\left(t-\tau_{i}(t)\right)\right| d t=\sum_{i=1}^{m} \int_{0}^{T} c_{i}\left(\gamma_{i}(s)\right)\left|x^{\prime}(s)\right| d s \leq \sum_{i=1}^{m} c_{i}^{u} \int_{0}^{T}\left|x^{\prime}(s)\right| d s
$$

Substituting (3.42) and (3.46) into (3.45) gives

$$
\begin{aligned}
\int_{0}^{T}\left|x^{\prime}(t)\right| d t & \\
\leq T & {\left[2 \bar{a}+\bar{\Gamma}|x|_{0}+\sum_{j=1}^{n} \bar{b}_{j}|x|_{0}\right]+\sum_{i=1}^{m} c_{i}^{u} \int_{0}^{T}\left|x^{\prime}(s)\right| d s } \\
\leq T & {\left[2 \bar{a}+\left(\bar{\Gamma}+\sum_{j=1}^{n} \bar{b}_{j}\right)\left(q+\int_{0}^{T}\left|x^{\prime}(t)\right| d t\right)\right]+\sum_{i=1}^{m} c_{i}^{u} \int_{0}^{T}\left|x^{\prime}(s)\right| d s } \\
\leq T & {\left[2 \bar{a}+\left(\bar{\Gamma}+\sum_{j=1}^{n} \bar{b}_{j}\right) q\right]+\left[T\left(\bar{\Gamma}+\sum_{j=1}^{n} \bar{b}_{j}\right)+\sum_{i=1}^{m} c_{i}^{u}\right] \int_{0}^{T}\left|x^{\prime}(t)\right| d t . }
\end{aligned}
$$


Since

$$
T\left(\bar{\Gamma}+\sum_{j=1}^{n} \bar{b}_{j}\right)+\sum_{i=1}^{m} c_{i}^{u}<1,
$$

we have

$$
\int_{0}^{T}\left|x^{\prime}(t)\right| d t<\frac{2 T \bar{a}+\left(\bar{\Gamma}+\sum_{j=1}^{n} \bar{b}_{j}\right) T q}{1-T\left(\bar{\Gamma}+\sum_{j=1}^{n} \bar{b}_{j}\right)-\sum_{i=1}^{m} c_{i}^{u}}:=p .
$$

Then,

$$
|x|_{0} \leq q+\int_{0}^{T}\left|x^{\prime}(t)\right| d t \leq q+p:=R_{0} .
$$

Again from (3.30), we get

$$
\left|x^{\prime}\right|_{0}<a^{u}+\sum_{j=1}^{n} b_{j}^{u}|x|_{0}+\sum_{i=1}^{m} c_{i}^{u}\left(1-\tau_{i}^{\prime}\right)^{u}\left|x^{\prime}\right|_{0}+h^{u} e^{R_{0}} .
$$

Since $\sum_{i=1}^{m} c_{i}^{u}\left(1-\tau_{i}^{\prime}\right)^{u}<1$, we have

$$
\left|x^{\prime}\right|_{0}<\frac{a^{u}+\sum_{j=1}^{n} b_{j}^{u} R_{0}+h^{u} e^{R_{0}}}{1-\sum_{i=1}^{m} c_{i}^{u}\left(1-\tau_{i}^{\prime}\right)^{u}}:=M_{0} .
$$

Choose $t_{M}, t_{m} \in[0, T]$, such that

$$
x\left(t_{M}\right)=\max _{t \in[0, T]} x(t), \quad x\left(t_{m}\right)=\min _{t \in[0, T]} x(t) .
$$

Then, it is clear that

$$
x^{\prime}\left(t_{M}\right)=0, \quad x^{\prime}\left(t_{m}\right)=0 .
$$

From this and (3.30), we obtain that

$$
\begin{aligned}
& a\left(t_{M}\right)=\sum_{j=1}^{n} b_{j}\left(t_{M}\right) x\left(t_{M}-\sigma_{j}\left(t_{M}\right)\right)+\sum_{i=1}^{m} c_{i}\left(t_{M}\right)\left(1-\tau_{i}^{\prime}\left(t_{M}\right)\right) x^{\prime}\left(t_{M}-\tau_{i}\left(t_{M}\right)\right)+\frac{h\left(t_{M}\right)}{e^{x\left(t_{M}\right)}}, \\
& a\left(t_{m}\right)=\sum_{j=1}^{n} b_{j}\left(t_{m}\right) x\left(t_{m}-\sigma_{j}\left(t_{m}\right)\right)+\sum_{i=1}^{m} c_{i}\left(t_{m}\right)\left(1-\tau_{i}^{\prime}\left(t_{m}\right)\right) x^{\prime}\left(t_{m}-\tau_{i}\left(t_{m}\right)\right)+\frac{h\left(t_{m}\right)}{e^{x\left(t_{m}\right)}} .
\end{aligned}
$$


It follows from (3.55) that

$$
a\left(t_{M}\right)-\sum_{i=1}^{m} c_{i}\left(t_{M}\right)\left(1-\tau_{i}^{\prime}\left(t_{M}\right)\right) M_{0}-\sum_{j=1}^{n} b_{j}\left(t_{M}\right) x\left(t_{M}\right)-\frac{h\left(t_{M}\right)}{e^{x\left(t_{M}\right)}} \leq 0,
$$

which implies

$$
\frac{a^{l}-\sum_{i=1}^{m} c_{i}^{u}\left(1-\tau_{i}^{\prime}\right)^{u} M_{0}}{\sum_{j=1}^{n} b_{j}^{u}}-x\left(t_{M}\right)-\frac{h^{u}}{\sum_{j=1}^{n} b_{j}^{l}} e^{-x\left(t_{M}\right)} \leq 0 .
$$

By the assertion (1) of Lemma 3.2, we have

$$
x\left(t_{M}\right) \leq u^{-} \quad \text { or } \quad x\left(t_{M}\right) \geq u^{+} .
$$

It follows from (3.56) that

$$
a\left(t_{m}\right)+\sum_{i=1}^{m} c_{i}\left(t_{m}\right)\left(1-\tau_{i}^{\prime}\left(t_{m}\right)\right) M_{0}-\sum_{j=1}^{n} b_{j}\left(t_{m}\right) x\left(t_{m}\right)-\frac{h\left(t_{m}\right)}{e^{x\left(t_{m}\right)}} \geq 0,
$$

which implies

$$
\frac{a^{u}+\sum_{i=1}^{m} c_{i}^{u}\left(1-\tau_{i}^{\prime}\right)^{u} M_{0}}{\sum_{j=1}^{n} b_{j}^{l}}-x\left(t_{m}\right)-\frac{h^{l}}{\sum_{j=1}^{n} b_{j}^{u}} e^{-x\left(t_{m}\right)} \geq 0 .
$$

By the assertion (3) of Lemma 3.2, we have

$$
l^{-} \leq x\left(t_{m}\right) \leq l^{+} .
$$

Hence, it follows from (3.50), (3.59), and (3.62) that

$$
\begin{gathered}
x\left(t_{M}\right) \in\left[l^{-}, u^{-}\right] \cup\left[u^{+}, R_{0}\right], \\
x\left(t_{m}\right) \in\left[l^{-}, l^{+}\right] .
\end{gathered}
$$

Clearly, $l^{ \pm}, u^{ \pm}$are independent of $\lambda$. Now, let us consider $Q N(x)$ with $x \in R$. Note that

$$
Q N(x)=\bar{a}-\sum_{j=1}^{n} \bar{b}_{j} x-\frac{\bar{h}}{e^{x}}
$$

It follows from the assertion (2) of Lemma 3.2 that $Q N(x)=0$ has two distinct solutions:

$$
\tilde{u}_{1}=x^{-}, \quad \tilde{u}_{2}=x^{+} .
$$


By the assertion (4) of Lemma 3.2, one can take $v^{-}, v^{+}>0$ such that

$$
u^{-}<v^{-}<v^{+}<u^{+}
$$

Let

$$
\begin{aligned}
& \Omega_{1}=\left\{x \in X \mid \begin{array}{l|l}
\max _{t \in[0, T]} x(t) \in\left(l^{-}-\delta, v^{-}\right), \\
\min _{t \in[0, T]} x(t) \in\left(l^{-}-\delta, l^{+}+\delta\right), \\
\max _{t \in[0, T]}\left|x^{\prime}(t)\right|<M_{0} .
\end{array}\right\}, \\
& \Omega_{2}=\left\{x \in X \mid \begin{array}{l|l}
\max _{t \in[0, T]} x(t) \in\left(v^{+}, R_{0}+\delta\right), \\
\min _{t \in[0, T]} x(t) \in\left(l^{-}-\delta, l^{+}+\delta\right), \\
\max _{t \in[0, T]}\left|x^{\prime}(t)\right|<M_{0} .
\end{array}\right\} .
\end{aligned}
$$

Then $\Omega_{1}, \Omega_{2}$ are bounded open subsets of X. Clearly, $\Omega_{i} \subset \Omega(i=1,2)$, where $\Omega$ as defined in Lemma 3.5. It follows from Lemma 3.5 that $N: \bar{\Omega}_{i} \rightarrow Z$ is a $k_{0}$-set-contractive map $(i=1,2)$. Therefore, it follows from Lemmas 2.1 and 3.3 that $N: \bar{\Omega}_{i} \rightarrow Z$ is $L$ - $k$-set contractive on $\bar{\Omega}_{i}(i=1,2)$ with $k=k_{0} / l(L) \leq k_{0}<1$.

By the assertion (4) of Lemma 3.2, (3.52), (3.63), (3.65), and (3.66), it is easy to verify that $\Omega_{i}$ satisfies the assumptions (i) and (ii) in Lemma $2.2(i=1,2)$. By the assertion (2) of Lemma 3.2, a direct computation gives

$$
\begin{gathered}
\operatorname{deg}_{B}\left\{J Q N, \Omega_{1} \cap \operatorname{Ker} L, 0\right\}=\operatorname{sgn}\left(-\sum_{j=1}^{n} \bar{b}_{j}+\frac{\bar{h}}{e^{x^{-}}}\right)=1, \\
\operatorname{deg}_{B}\left\{J Q N, \Omega_{2} \cap \operatorname{Ker} L, 0\right\}=\operatorname{sgn}\left(-\sum_{j=1}^{n} \bar{b}_{j}+\frac{\bar{h}}{e^{x^{+}}}\right)=-1 .
\end{gathered}
$$

Here, $J$ is taken as the identity mapping since $\operatorname{Im} Q=\operatorname{Ker} L$. So far we have proved that $\Omega_{i}$ satisfies all the assumptions in Lemma $2.2(i=1,2)$. Hence, (3.1) has at least two $T$-periodic solutions $x_{i}^{*}(t)$ and $x_{i}^{*} \in \operatorname{dom} L \cap \bar{\Omega}_{i}(i=1,2)$. Since $\bar{\Omega}_{1} \cap \bar{\Omega}_{2}=\emptyset, x_{i}^{*}(i=1,2)$ are different. Let $y_{i}^{*}(t)=e^{x_{i}^{*}(t)}(i=1,2)$. Then $y_{i}^{*}(t)(i=1,2)$ are two different positive $T$-periodic solutions of (1.1). The proof is complete.

Example 3.7. Consider the following equation:

$$
\frac{d y(t)}{d t}=y(t)\left[a(t)-b(t) \ln y(t-\sigma(t))-c(t) \frac{d}{d t} \ln y(t-\tau(t))\right]-h(t),
$$


where

$$
\begin{gathered}
a(t)=\left(\frac{1}{4.2 e}+\frac{1}{4.2 e} \sin ^{2} t\right) \epsilon, \quad b(t)=(2.35+0.15 \sin t) \epsilon, \\
c(t)=(0.9+0.1 \cos t) \epsilon, \quad \sigma(t) \equiv 2 \pi, \quad \tau(t) \equiv 4 \pi, \quad h=\frac{3 \epsilon}{2 e}+\frac{\epsilon}{2 e} \sin t,
\end{gathered}
$$

and the constant $\epsilon>0$. Clearly, $\left(H_{1}\right)$ is satisfied.

Let $\mu(t)$ be the inverse function of $t-\sigma(t)$, and $\gamma(t)$ be the inverse function of $t-\tau(t)$. Then we have

$$
\begin{gathered}
\mu(t)=t+2 \pi, \quad \gamma(t)=t+4 \pi, \\
\Gamma(t)=\frac{b(\mu(t))}{1-\sigma^{\prime}(\mu(t))}-\frac{c^{\prime}(\gamma(t))}{1-\tau^{\prime}(\gamma(t))}=b(t+2 \pi)-c^{\prime}(t+4 \pi)=(2.35+0.25 \sin t) \epsilon .
\end{gathered}
$$

Hence, $\left(H_{2}\right)$ is satisfied.

It is easy to see that

$$
\begin{gathered}
T=2 \pi, \quad a^{u}=\frac{\epsilon}{2.1 e}, \quad a^{l}=\frac{\epsilon}{4.2 e}, \quad \bar{a}=\frac{\epsilon}{2.8 e}, \quad b^{u}=2.5 \epsilon, \quad b^{l}=2.2 \epsilon, \quad \bar{b}=2.35 \epsilon, \\
\Gamma^{u}=2.6 \epsilon, \quad \Gamma^{l}=2.1 \epsilon, \quad \bar{\Gamma}=2.35 \epsilon, \quad c^{u}=\epsilon, \quad h^{u}=\frac{2 \epsilon}{e}, \quad h^{l}=\frac{\epsilon}{e}, \quad \bar{h}=\frac{3 \epsilon}{2 e} .
\end{gathered}
$$

Therefore, we have

$$
\begin{gathered}
\frac{h^{l}}{\Gamma^{u}}=\frac{1}{2.6 e^{\prime}}, \quad \frac{a^{u}}{\Gamma^{l}}=\frac{1}{4.41 e^{\prime}} \\
1+\ln \frac{h^{l}}{\Gamma^{u}}=-\ln 2.6 .
\end{gathered}
$$

So,

$$
1+\ln \frac{h^{l}}{\Gamma^{u}}<\frac{a^{u}}{\Gamma^{l}}<\frac{h^{l}}{\Gamma^{u}}<1
$$

Hence, $\left(H_{3}\right)$ is satisfied. 
Also, it is easy to see that

$$
\begin{gathered}
\frac{a^{l}-c^{u}\left(1-\tau^{\prime}\right)^{u} M_{0}}{b^{u}}=\frac{\epsilon /(4.2 e)-\epsilon M_{0}}{2.5 \epsilon}=\frac{1}{10.5 e}-\frac{M_{0}}{2.5}<1, \\
q=2\left|\ln \frac{h^{l}}{\Gamma^{u}}\right|=2(\ln 2.6+1)>1, \quad p=\frac{2 T \bar{a}+(\bar{\Gamma}+\bar{b}) T q}{1-T(\bar{\Gamma}+\bar{b})-c^{u}}=\frac{\pi \epsilon /(0.7 e)+18.8(\ln 2.6+1) \pi \epsilon}{1-9.4 \pi \epsilon-\epsilon}, \\
R_{0}=q+p, \\
M_{0}=\frac{a^{u}+b^{u} R_{0}+h^{u} e^{R_{0}}}{1-c^{u}\left(1-\tau^{\prime}\right)^{u}}=\frac{\epsilon /(2.1 e)+2.5 \epsilon R_{0}+2 \epsilon e^{R_{0}-1}}{1-\epsilon} .
\end{gathered}
$$

Therefore, we obtain that

$$
R_{0}>q>\frac{a^{l}-c^{u}\left(1-\tau^{\prime}\right)^{u} M_{0}}{b^{u}} .
$$

Noticing that $\lim _{\epsilon \rightarrow 0^{+}} R_{0}=q$, we have $\lim _{\epsilon \rightarrow 0^{+}} M_{0}=0$. Therefore, for some sufficiently small $\epsilon>0$, the following inequalities hold:

$$
\begin{gathered}
\max \left\{1+\ln \frac{h^{u}}{b^{l}}, 0\right\}=\max \{-\ln 1.1,0\}=0<\frac{a^{l}-c^{u}\left(1-\tau^{\prime}\right)^{u} M_{0}}{b^{u}}, \\
T(\bar{\Gamma}+\bar{b})+c^{u}=9.4 \pi \epsilon+\epsilon<1 .
\end{gathered}
$$

By (3.76)-(3.77), $\left(H_{4}\right)$ is also satisfied. Therefore, all necessary conditions of Theorem 3.6 are satisfied. By Theorem 3.6, (3.69) has at least two positive $2 \pi$-periodic solutions.

\section{Acknowledgment}

This paper is supported by the National Natural Science Foundation of China (Grant nos. 10971085 and 11061016).

\section{References}

[1] R. Wang and X. Zhang, "Positive periodic solution for a neutral logarithmic population model with feedback control," Applied Mathematics and Computation, vol. 217, no. 19, pp. 7692-7702, 2011.

[2] S. Lu and W. Ge, "Existence of positive periodic solutions for neutral logarithmic population model with multiple delays," Journal of Computational and Applied Mathematics, vol. 166, no. 2, pp. 371-383, 2004.

[3] Q. Wang, Y. Wang, and B. Dai, "Existence and uniqueness of positive periodic solutions for a neutral logarithmic population model," Applied Mathematics and Computation, vol. 213, no. 1, pp. 137-147, 2009.

[4] M. Tang and X. Tang, "Positive periodic solutions for neutral multi-delay logarithmic population model," Journal of Inequalities and Applications, vol. 2012, pp. 1-10, 2012. 
[5] Y. Luo and Z. Luo, "Existence of positive periodic solutions for neutral multi-delay logarithmic population model," Applied Mathematics and Computation, vol. 216, no. 4, pp. 1310-1315, 2010.

[6] Y. Xia, "Almost periodic solution of a population model: via spectral radius of matrix," Bulletin of the Malaysian Mathematical Sciences Society. In press.

[7] Y. K. Li, "On a periodic neutral delay logarithmic population model," Journal of Systems Science and Mathematical Sciences, vol. 19, no. 1, pp. 34-38, 1999.

[8] Y. Chen, "Multiple periodic solutions of delayed predator-prey systems with type IV functional responses," Nonlinear Analysis: Real World Applications, vol. 5, no. 1, pp. 45-53, 2004.

[9] G. Hetzer, "Some remarks on $\Phi_{+}$-operators and on the coincidence degree for a Fredholm equation with noncompact nonlinear perturbations," Annales de la Societé Scientifique de Bruxelles, vol. 89, no. 4, pp. 497-508, 1975.

[10] G. Hetzer, "Some applications of the coincidence degree for set-contractions to functional differential equations of neutral type," Commentationes Mathematicae Universitatis Carolinae, vol. 16, no. 1, pp. 121$138,1975$.

[11] R. E. Gaines and J. L. Mawhin, Coincidence Degree, and Nonlinear Differential Equations, Springer, Berlin, Germany, 1977.

[12] Z. Liu and Y. Mao, "Existence theorem for periodic solutions of higher order nonlinear differential equations," Journal of Mathematical Analysis and Applications, vol. 216, no. 2, pp. 481-490, 1997. 


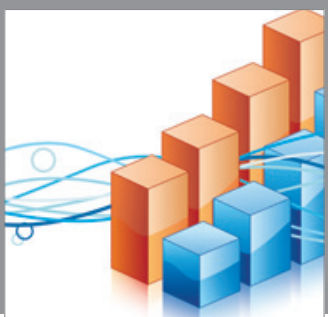

Advances in

Operations Research

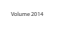

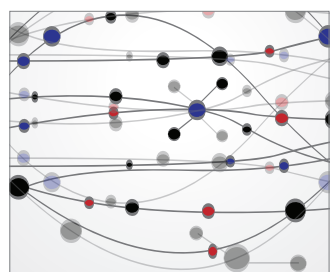

\section{The Scientific} World Journal
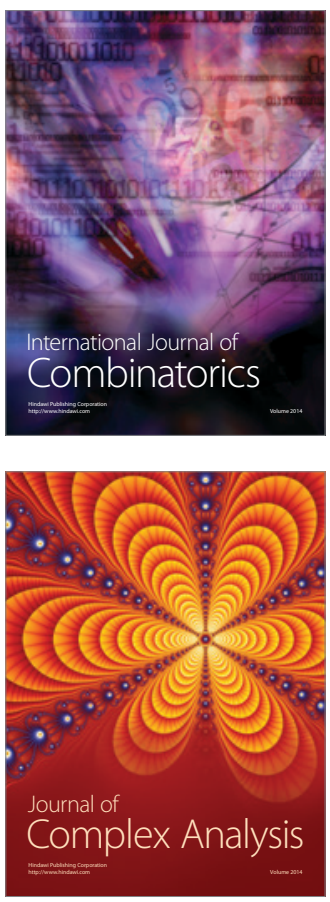

International Journal of

Mathematics and

Mathematical

Sciences
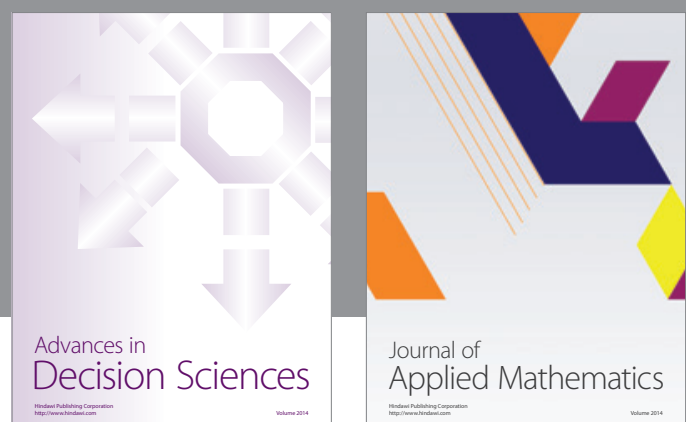

Journal of

Applied Mathematics
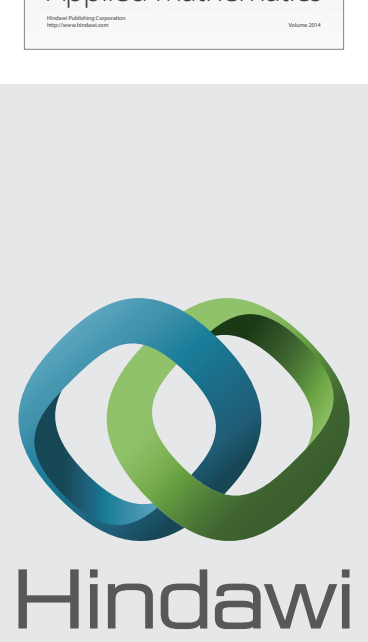

Submit your manuscripts at http://www.hindawi.com
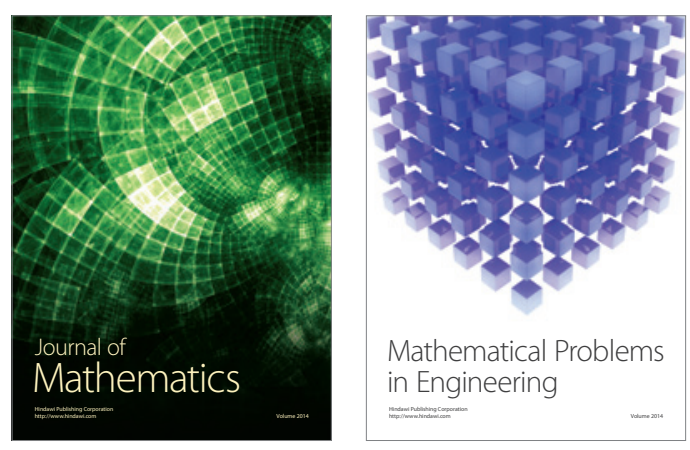

Mathematical Problems in Engineering
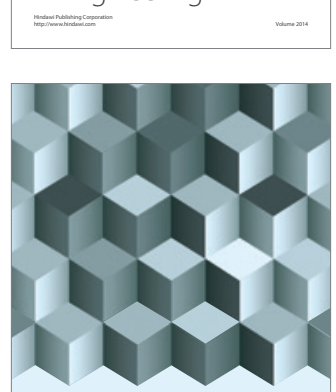

Journal of

Function Spaces
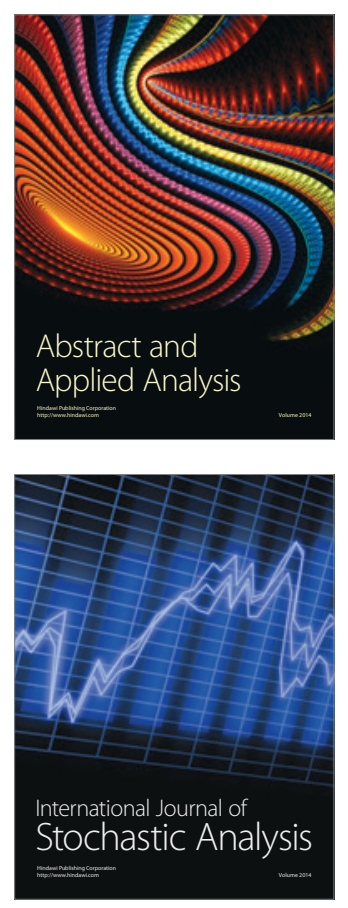

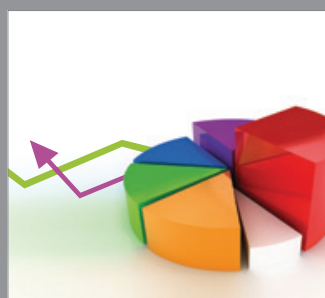

ournal of

Probability and Statistics

Promensencen
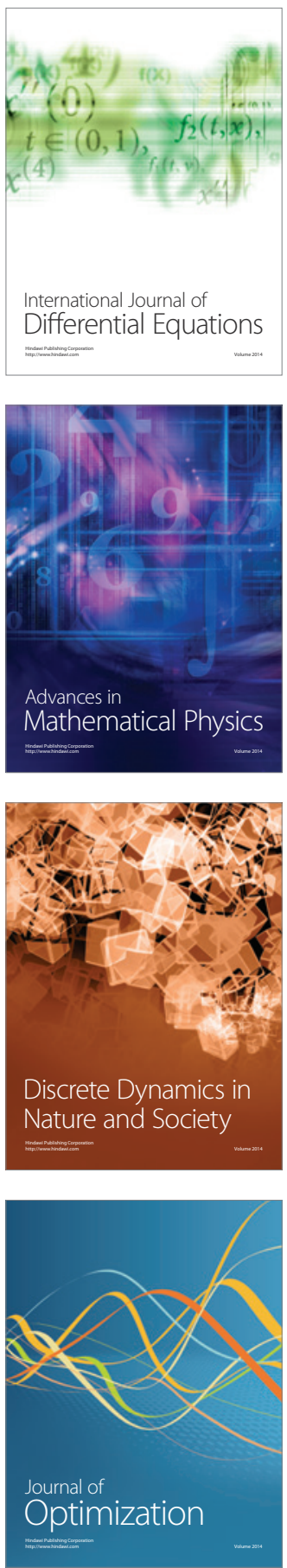